\title{
THE AREA DISTRIBUTION OF SOLAR MAGNETIC BRIGHT POINTS
}

\author{
P. J. Crockett ${ }^{1}$, M. Mathioudakis ${ }^{1}$, D. B. Jess ${ }^{1}$, S. Shelyag ${ }^{1}$, F. P. Keenan ${ }^{1}$, and D. J. Christian ${ }^{2}$ \\ ${ }^{1}$ Astrophysics Research Centre, School of Mathematics and Physics, Queen's University, Belfast BT7 1NN, UK \\ ${ }^{2}$ Department of Physics and Astronomy, California State University, Northridge, CA 91330, USA \\ Received 2010 May 14; accepted 2010 September 10; published 2010 October 1
}

\begin{abstract}
Magnetic bright points (MBPs) are among the smallest observable objects on the solar photosphere. A combination of $G$-band observations and numerical simulations is used to determine their area distribution. An automatic detection algorithm, employing one-dimensional intensity profiling, is utilized to identify these structures in the observed and simulated data sets. Both distributions peak at an area of $\approx 45,000 \mathrm{~km}^{2}$, with a sharp decrease toward smaller areas. The distributions conform with log-normal statistics, which suggests that flux fragmentation dominates over flux convergence. Radiative magneto-convection simulations indicate an independence in the MBP area distribution for differing magnetic flux densities. The most commonly occurring bright point size corresponds to the typical width of inter-granular lanes.
\end{abstract}

Key words: Sun: evolution - Sun: granulation - Sun: photosphere - Sun: surface magnetism

\section{INTRODUCTION}

The dominant pattern covering the quiet solar photosphere is the granulation. Plasma flows remove the magnetic flux from granules into the dark inter-granular lanes where it clusters together to form small magnetic concentrations of $1^{\prime \prime}$ or less, with field strengths often in excess of a kilogauss. Magnetic bright points (MBPs) are a manifestation of these kilogauss fields. They are among the smallest observable objects on the photosphere appearing as intensity enhancements within the inter-granular lanes. They are formed by complex processes involving the interaction of magnetic fields with the convectively unstable plasma and can provide a conduit for channeling kinetic energy into the upper atmosphere (de Wijn et al. 2009). Although all MBPs are found to reside in areas of high magnetic field, the opposite is not always true, with some high magnetic field in plage regions appearing devoid of MBPs (Ishikawa et al. 2007). An examination of the physical parameters required for an MBP to exist, i.e., minimum or maximum size, and lower magnetic field threshold, will further our knowledge on the creation and evolution of such small-scale photospheric magnetic fields.

The physical processes responsible for MBP formation have been simulated by Schüssler et al. (2003) and Shelyag et al. (2004). Magneto-convection models for convectively unstable plasma in the photosphere are combined with the radiative properties of that plasma and include the partial ionization of hydrogen and the other most abundant elements. The validation of numerical simulations by observations may allow us to conclude whether the physics in the simulations can describe the real Sun and hence help us interpret the observational findings.

$G$-band imaging forms a common basis for MBP observations. Sánchez Almeida et al. (2004) find that MBPs have a maximum intensity 1.8 times the mean photospheric value. Several authors propose that such intensity enhancements are caused by a significant weakening of the $\mathrm{CH}$ absorption lines, due to the dissociation of $\mathrm{CH}$ molecules at increasing temperatures (Sánchez Almeida et al. 2001; Steiner et al. 2001; Langhans et al. 2002). The latter group identify two types of bright points that occur in $G$-band images; those that are associated with magnetic structures, and others that exist at the edge of rapidly expanding granules. The latter are believed to be density en- hancements, caused by a buildup of material attempting to flow down the thin inter-granular lanes at the perimeter of a granule.

The sheer number of MBPs requires automated algorithms for their detection and tracking. In general, higher spatial resolution leads to smaller sizes being detected. Berger et al. (1995) applied an altered blob-finding algorithm to separate MBPs from granules. They quantified the MBP size in terms of the FWHM intensity diameter, taking the smallest dimension across the identified objects. A semi-automatic process was implemented that included nonlinear least-squares Gaussian fitting to the observed intensity profiles. Their analysis revealed a modal diameter of $220 \mathrm{~km}$, an average diameter of $250 \mathrm{~km}$, and a diameter range of 120-600 km. Bovelet \& Wiehr (2003) employed a specialized version of the multiple-level tracking pattern recognition software (Bovelet \& Wiehr 2001), which applies several decreasing intensity thresholds to an image. A dominant diameter of $220 \pm 25 \mathrm{~km}$ was detected. Wiehr et al. (2004) repeated this procedure using higher spatial resolution observations and found a predominant diameter of $160 \pm$ 20 km. Sánchez Almeida et al. (2004) visually identified MBPs in individual $G$-band images. The area was determined by a segmentation algorithm, and their diameter was measured by fitting a double Gaussian profile. They obtain $135 \mathrm{~km}$ for the minor axis, which seems to be set by the angular resolution of the observations. This double Gaussian decontaminates the profiles, by taking into account the intensity distribution of the dark local background, within which the MBPs are situated (Title \& Berger 1996). Utz et al. (2009) utilized an altered version of the Bovelet \& Wiehr (2001) algorithm and applied it to Hinode Solar Optical Telescope observations. The size of MBPs was defined by placing an upper and lower intensity threshold on the segmented structures, resulting in mean diameters of $166-218 \mathrm{~km}$.

In this Letter, we use observations and numerical simulations to investigate the area distribution of MBPs. An automatic detection and a tracking algorithm, described in (Crockett et al. 2009, hereafter Paper I), are developed further and applied to high resolution $G$-band images. Section 2 discusses the observations, with emphasis on an automated algorithm used for MBP detection and size determination. A description of the numerical simulations is given in Section 3. Our main findings are presented in Section 4, with concluding remarks in Section 5. 

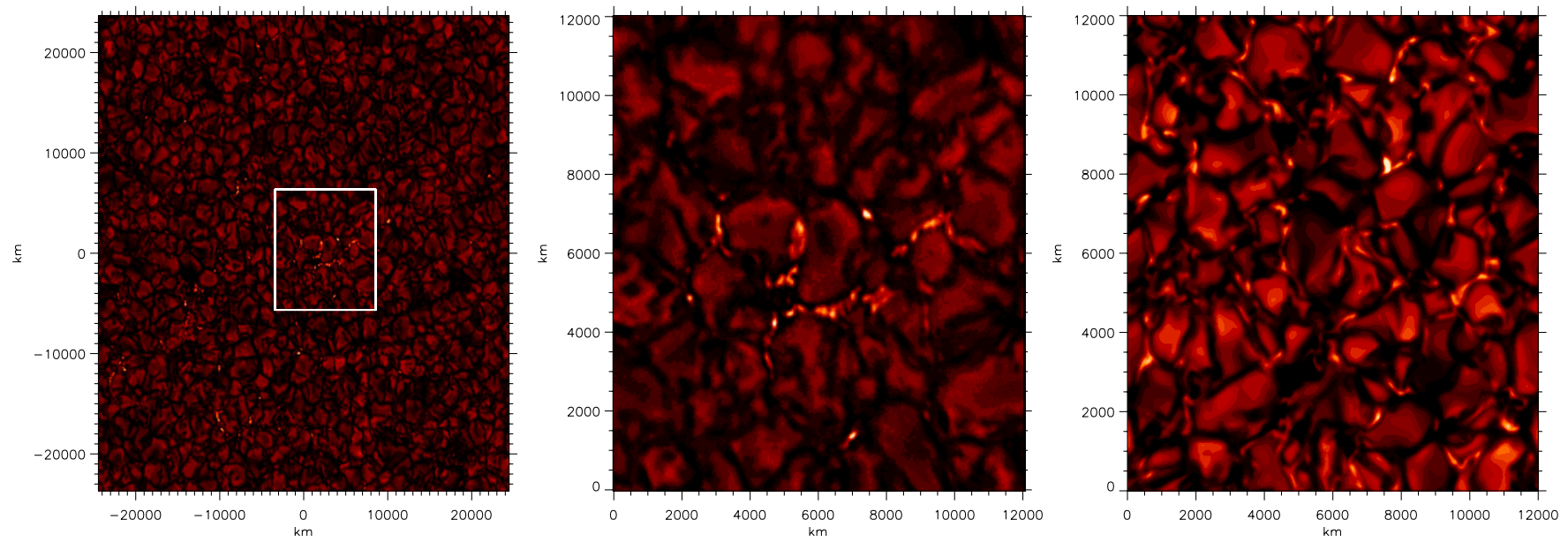

Figure 1. Left: a $70^{\prime \prime} \times 70^{\prime \prime} G$-band image from the ROSA data set. Middle: a $12 \times 12 \mathrm{Mm}$ section of the $G$-band image. Right: $G$-band simulation for an average field of $200 \mathrm{G}$

\section{OBSERVATIONS AND DATA ANALYSIS}

The data were obtained by the newly commissioned Rapid Oscillations in the Solar Atmosphere (ROSA) instrument, installed at the $76 \mathrm{~cm}$ Dunn Solar Telescope (DST), in New Mexico, USA (Jess et al. 2010). The observations were taken on 2009 May 28 through a $12 \AA$ filter, centered at $4305 \AA$ ( $G$ band), during a period of excellent seeing. Post-facto speckle reconstruction algorithms (Wöger et al. 2008), in addition to rigorous image de-stretching using a $40 \times 40$ grid (equating to a $\approx 1^{\prime \prime} 7$ separation between spatial samples (Jess et al. 2008)), were implemented to remove effects caused by atmospheric seeing. We observed a $70^{\prime \prime} \times 70^{\prime \prime}$ quiet solar region at the disk center, achieving diffraction-limited imaging with 0.069 pixel $^{-1}$. Figure 1 displays a typical $G$-band image from the data set, with multiple MBPs visible in the central region. Analysis of the data was performed with an updated version of a detection algorithm described in Paper I, which uses intensity thresholding to map the inter-granular lanes. A compass search allows MBPs to be disentangled from bright pixels within granules, while object growing accounts for any pixels that might have been removed when mapping the lanes. One of the disadvantages of the algorithm described in Paper I is the requirement for the image to be divided into segments, with each subsequent segment being processed individually. Here, we use an updated algorithm which operates on the entire $70^{\prime \prime} \times 70^{\prime \prime}$ image sequence, thus improving computational time and accuracy. This development is particularly important, as it permits accurate estimates of MBP areas. Mapping the location of the inter-granular lanes, with an overestimation of the intensity threshold, is used to separate out bright objects. The threshold set is the mean image intensity plus $1 \sigma$. All structures under this level are considered a lane and are not investigated by our algorithm. The vast majority of MBPs retain higher intensities, however some very dull MBPs may be lost at this stage. Objects are then investigated individually. We impose a $3 \sigma$ intensity variation limit on each object, in order to fully separate MBPs from the granules. Any bright object possessing an intensity range greater than $3 \sigma$ is broken up into smaller objects, until the resulting structures comply with this condition.

The detection of MBPs is carried out by an extended version of the compass search (see Paper I, Section 4.3), and incorporates gradient thresholding through intensity profiling. A onedimensional variation in intensity, across a selected region of the image, is first determined (see the left panel of Figure 2). Intensity profiles for each object are established for eight directions, symmetrically positioned about the objects' center of gravity. The stipulation that a lane must be in close proximity to the MBP remains. The algorithm now actively searches for inter-granular lanes, by using the turning points of the intensity profiles, which are located at the center of the lanes (left panel of Figure 2). Hence, the lanes are located from in situ intensity profiling. Each measurement is specific, not only to individual objects, but in every considered direction as well. To establish each turning point the one-dimensional line, from which intensity profiles are procured, is extended until two stationary points exist in the profile, i.e., where the rate of change in intensity $(y)$ as a function of distance $(x)$ is zero, $d y / d x=0$. A limit on the distance between the turning points eliminates large objects, such as granules.

Gradient thresholding is applied to all intensity profiles to disentangle MBPs from granules. MBPs retain a very steep intensity change in all directions, compared to a more gradual variation associated with granules. The maximum gradient is determined from any part of a profile falling between the two turning points (see Figure 2). The threshold gradient is derived for each individual image through a selection of 500 random objects and is calculated prior to the execution of the algorithm. A threshold is determined by adding a $1 \sigma$ value to the median gradient recorded for each image.

A significant improvement of the present algorithm concerns the growing of MBPs. A newly developed process provides an independent threshold range for each object for accurate area representation. The algorithm rotates a one-dimensional line through $360 \mathrm{deg}$, in 5 deg steps, about an object's center of gravity. Intensity values at the turning points of the profiles, i.e., the lanes, are acquired at each angle. To aid the accurate determination of turning points, the data are re-binned by a factor of 10 and smoothed. Thus, a narrow inter-granular lane and the associated turning point can be clearly identified. The maximum turning point intensity is taken to provide a lower cutoff to our growing algorithm, while the upper boundary is set as the maximum intensity level occurring within the seed region. The growing procedure includes any conjoining pixels that are above the lower threshold cutoff. The MBP area is determined by totaling the number of pixels within each structure. Our sampling of $0.069 \mathrm{pixel}^{-1}$ provides an area of $2500 \mathrm{~km}^{2}$ pixel $^{-1}$. This procedure, demonstrated in Figure 3, reproduces $90 \%$ of 

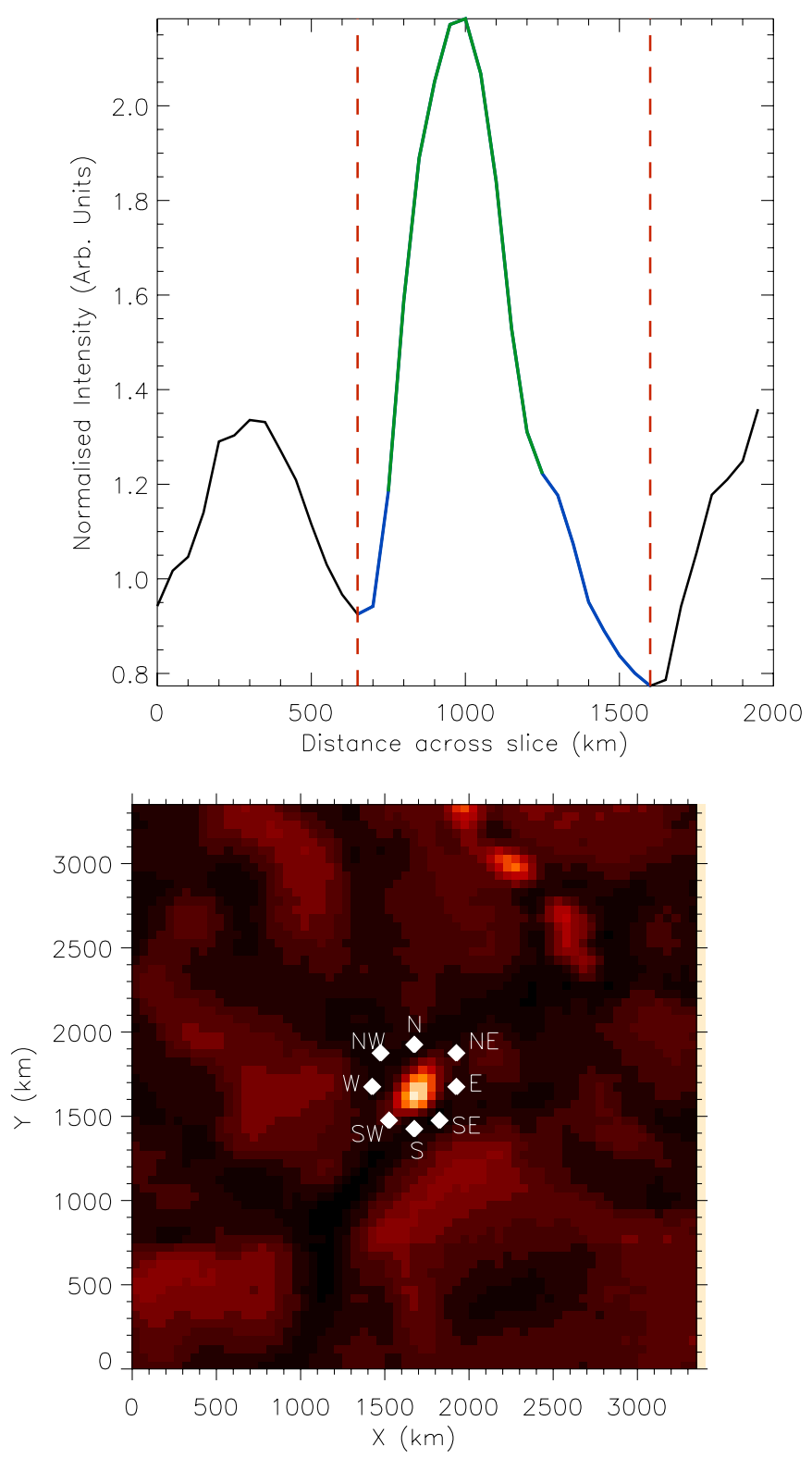

Figure 2. Top: intensity profile typical of an MBP. A steep intensity gradient is followed by two minimum turning points at the center of the dark lane. The algorithm detects the minimum turning points, marked by dashed red lines, in eight separate directions. Bottom: the white marks indicate the locations of turning points in the image. These turning points reside in the center of the inter-granular lanes. The MBP shown has an area of $127,500 \mathrm{~km}^{2}$.

MBPs to within a $10 \%$ error of visually identified areas. Setting the lower intensity threshold as the brightest, surrounding lane enforces an upper limit on the area of the MBPs, i.e., MBPs are grown to their maximum dimensions.

\section{NUMERICAL SIMULATIONS}

We use the MURaM code (Vögler et al. 2005) to carry out simulations of radiative magneto-convection in the upper solar convection zone and photosphere. This code uses a fourthorder, central difference scheme for computing the spatial derivatives, and a fourth-order, Runge-Kutta scheme to advance the solution forward in time. The solution is stabilized against numerical instabilities using additional artificial hyperdiffusive terms, described in detail by Caunt \& Korpi (2001), Vögler et al. (2005), and Shelyag et al. (2008). The size of the computational domain used for the simulations is $12 \times 12 \times 1.4 \mathrm{Mm}^{3}$, resolved by $480 \times 480 \times 100$ grid cells providing a resolution of $25 \mathrm{~km}$ per grid cell. However, we emphasize that as a result of hyperdiffusivity, the size of the smallest structures produced in the simulations can be larger than a single grid cell. Due to the dependence of the hyperdiffusivity coefficients on the local solution, it is not possible to globally define a quantity, uniquely representing the resolution. However, a standard test, such as the strong (compression ratio 100) Riemann shock tube (Sod 1978), can be used to provide an indication of the resolution. The results of such tests for similar codes (i.e., fourth- to sixth-order central difference spatial scheme and hyperdiffusive sources) show that even for such an extreme case, the shock front is diffused over 2-4 grid cells, depending on the relative position of the shock front with respect to the grid (Caunt \& Korpi 2001; Shelyag et al. 2008). Consequently, the resolution of the code for this case is about $50-100 \mathrm{~km}$, a value similar to the resolution of the observations. The side boundaries are periodic, the upper boundary is closed for vertical and stressfree horizontal plasma motions, while the bottom boundary is transparent. The level corresponding to the visible solar surface is located approximately $400 \mathrm{~km}$ below the upper boundary. This setup allows us to perform radiative diagnostics of $G$-band images and directly compare them with the observations. A detailed description of the method used is given in Shelyag et al. (2004). Here, we provide a brief description of the process.

For each of the light rays corresponding to a vertical plasma column in the simulation, we compute the LTE spectrum in the 4295-4315 $\AA$ range, which consists of 328 absorption lines, 239 of which are produced by $\mathrm{CH}$ molecules. The calculated spectrum is convolved with the $G$-band filter function. The magnetic splitting of $\mathrm{CH}$ lines and its influence on $G$-band intensities are sufficiently small for this effect to be neglected. $G$-band images obtained using this technique reproduce the dynamic and radiative properties of magneto-convection and show a large number of $G$-band bright points, corresponding to the heated, and partially evacuated, magnetic flux tubes seen in Figure 1.

\section{RESULTS}

A series of 500 images was investigated, incorporating a total of 63,312 MBPs. The MBPs cover approximately $0.42 \%$ of the solar surface with a variance between $0.33 \%$ and $0.53 \%$ across the time series. Figure 4 displays the area distribution of MBPs, with their occurrence normalized to the mean number detected across all bins. The distribution was created by a summation of MBPs across all images, in 1 pixel bins. This technique may lead to the "double counting" of MBPs, some of which may have longer lifetimes than others. However, snapshots of single frames produce a similar distribution, with approximately the same peak. Therefore, this technique is equivalent to the summation of multiple snapshot distributions, each with similar parameters, resulting in an overall identical distribution. We therefore believe that any double counting does not pose a problem in our interpretation.

The nature of the distribution appears to conform with lognormal statistics. To confirm this, a log-normal probability density function (PDF) of the form,

$$
\mathrm{PDF}_{\log -\text { normal }}=\frac{1}{x \mu \sqrt{2 \pi}} \exp \frac{-(\ln x-\mu)^{2}}{2 \sigma^{2}}
$$



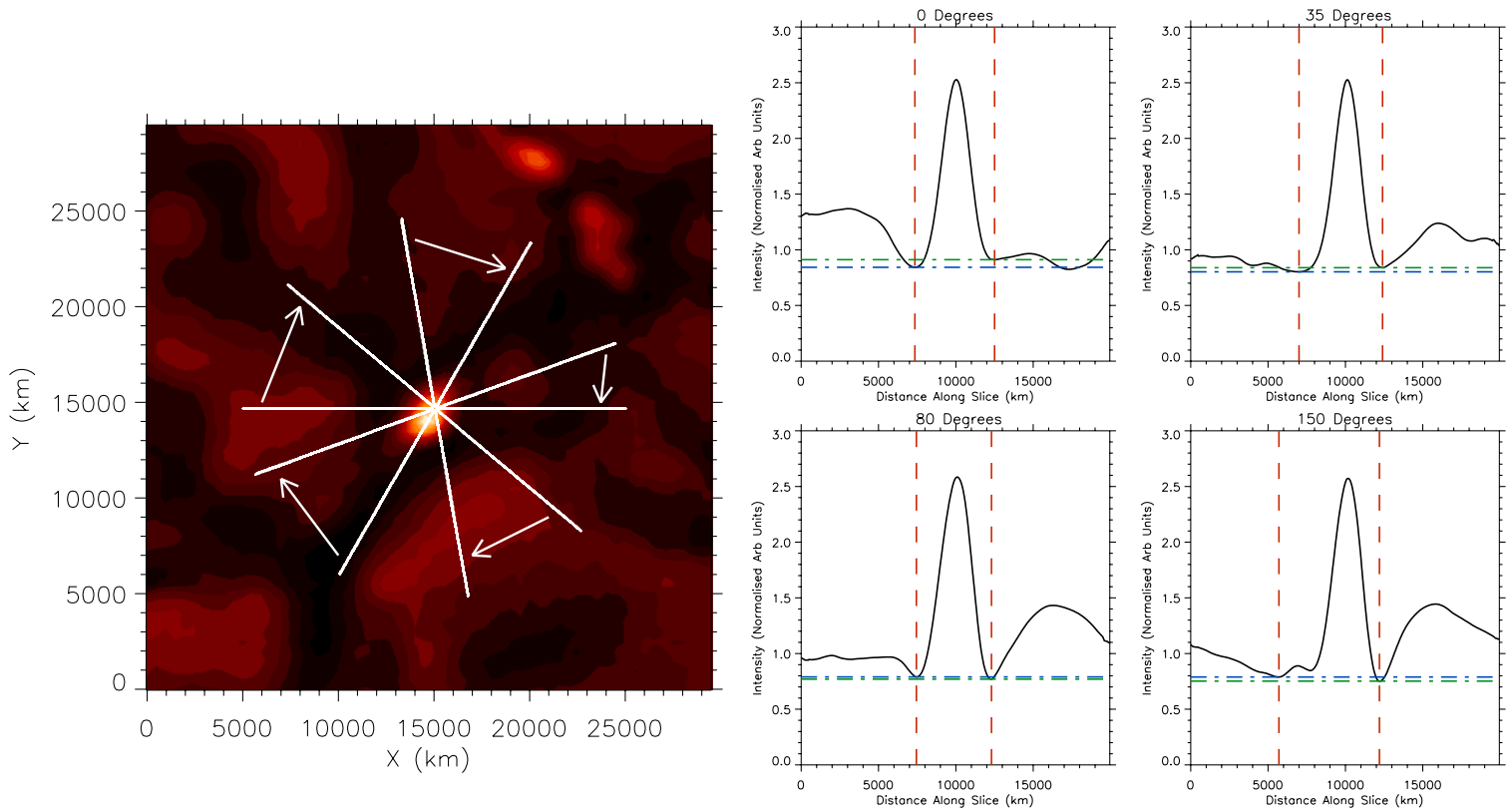

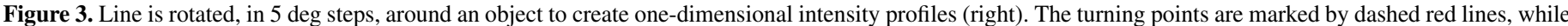

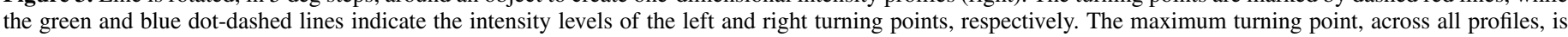
imposed as the lower threshold for MBP growing. Note the re-binning of the data to ensure that turning points are located accurately.

where $\mu$ and $\sigma$ are, respectively, the mean and standard deviation of $\ln x$, is fitted to the data. Values of $\mu=3.25$ and $\sigma=0.65$ produce an excellent fit, shown by the overplotted solid red line in Figure 4. To quantify the goodness of the fit, the $\chi^{2}$ error statistic (Wall \& Jenkins 2003) of the form,

$$
\chi^{2}=\sum_{i=1}^{n} \frac{\left(O_{i}-E_{i}\right)^{2}}{E_{i}},
$$

where $O_{i}$ and $E_{i}$ are, respectively, the observed and expected frequencies, is utilized. The observed frequencies correspond to the values obtained in the data, while the expected frequencies correspond to the theoretical values set by the log-normal fit. Comparison of the real data with the fitted distribution reveals a conformity of $99.5 \%$ and confirms that the MBP area distribution is well described by log-normal statistics.

The peak of the distribution occurs at an area of $45,000 \mathrm{~km}^{2}$. Assuming a circular geometry, this corresponds to a diameter of $230 \mathrm{~km}$. While this estimate appears in general agreement with earlier works, there are a number of points that need to be emphasized. Utz et al. (2009) find diameters of $218 \pm$ $48 \mathrm{~km}$ using a spatial sampling of 0.108 pixel $^{-1}$ on Hinode SOT, but this is dependent on the spatial sampling. Reducing the spatial sampling to $0^{\prime \prime} 054$ pixel $^{-1}$ gives a diameter of $166 \pm 31 \mathrm{~km}$. The latter value is in agreement with the results of Wiehr et al. (2004). Differences in the diameters may be explained by differences in the detection algorithms employed. Wiehr et al. (2004) employ the Multi-Level Tracking (MLT) algorithm which utilizes decreasing intensity levels to identify and separate objects. MLT sets an initial uppermost intensity level. Bright structures which exceed this threshold are tagged. The intensity level is then lowered. Pixels above the new intensity level, adjacent to the structures identified in the previous level, are added. New structures that appear at this level are tagged separately. This repetitive procedure is terminated after a last extension to a final intensity level deemed adequate for representing the observed pattern. The structures are forced to be separated by 2 pixels on all sides. The enforced separation and the somewhat arbitrary final threshold level may affect the dimensions of the MBPs measured by missing dim edge pixels. Utz et al. (2009) employ a similar repetitive intensity thresholding technique to separate granules and MBPs. They impose an upper and lower intensity boundary on the pixels of an object to determine its size. The upper boundary is given by the maximum intensity in the object while the lower boundary is defined as the maximum minus $30 \%$ of the mean photospheric intensity. Again these conditions may limit the final size of MBPs to the brightest pixels. This effect may be exaggerated at the higher spatial sampling. Instead our algorithm sets a threshold which is specific to each MBP. Moreover, by taking the threshold as the highest intensity within the lane, we determine an upper limit in the MBP area including dim edge pixels which may be neglected in the earlier studies.

In Figure 4, the vertical dashed line at $10,000 \mathrm{~km}^{2}$ marks a 4 pixel $(2 \times 2)$ resolution threshold, thus placing a limit on the smallest structures that can be resolved in our observations. The procedure of identifying the smallest structures in the observations was tested by convolving the simulated $G$-band images with the Airy function corresponding to a $76 \mathrm{~cm}$ aperture and re-binning them to the spatial sampling of the observations. This test showed that 11 out of 12 MBPs, each covering an area of 3-5 pixels in the degraded images, correspond to MBPs in the original (non-degraded) images. The sharp drop in the area of MBPs below the peak implies that underlying physical processes are limiting the creation, and evolution, of very small structures. In addition, the distribution shows only a relatively small number of large-scale MBPs with area greater than $200,000 \mathrm{~km}^{2}$.

Errors in the distribution were determined by comparing the visual estimates of the area with the output of the algorithm. The comparison reveals the distribution of errors for each bin from which a sigma value was determined. At the peak of our distribution the error is $\pm 8 \%( \pm 3600 \mathrm{~km})$. The uncertainty at the high end of the distribution decreases while as we approach the diffraction limit the errors become larger due to small number statistics. 

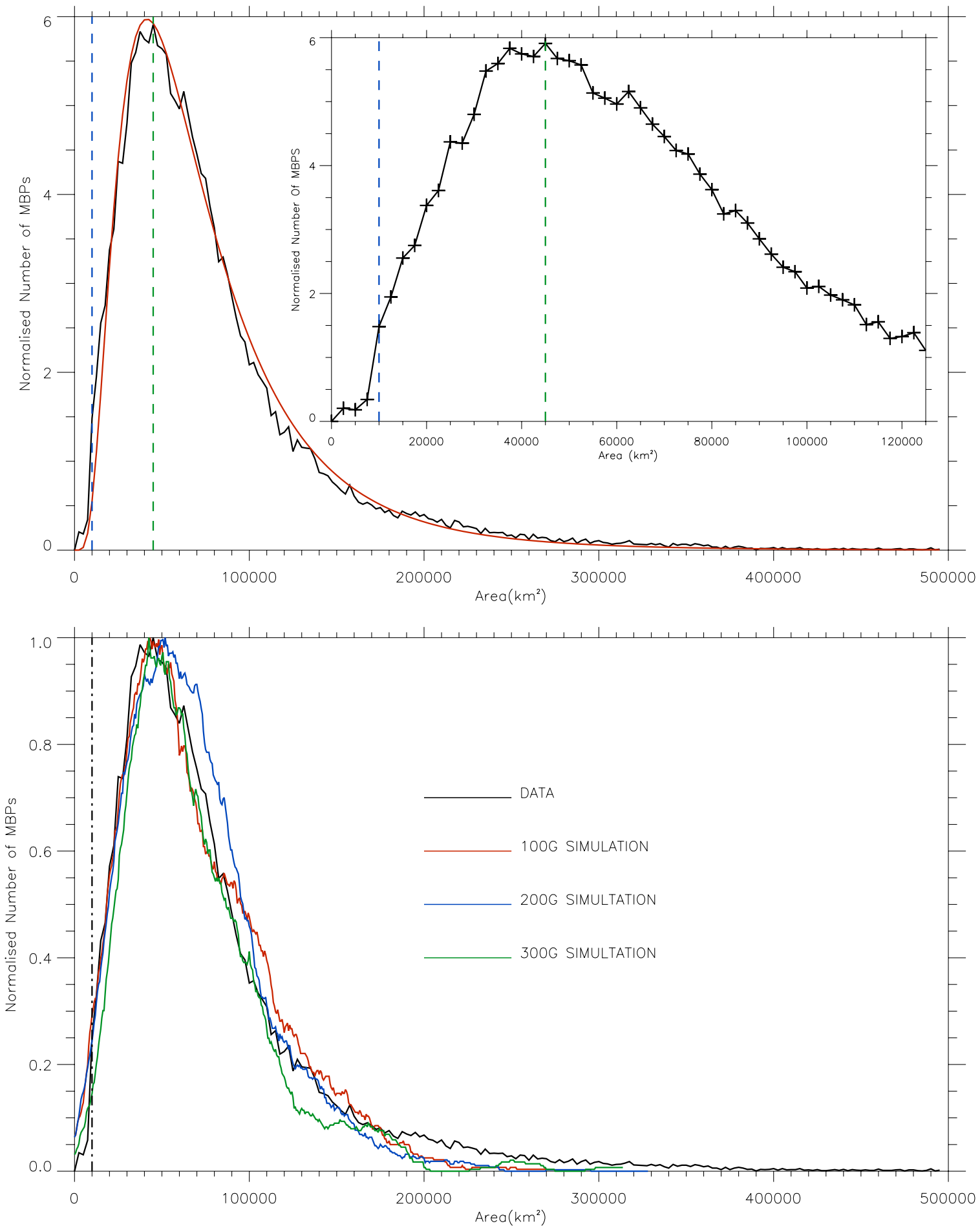

Figure 4. Top: the observed area distribution of MPBs. The green and blue dashed lines mark, respectively, the peak of the distribution at $45,000 \mathrm{~km}^{2}$ and the diffraction limit at $10,000 \mathrm{~km}^{2}$. A log-normal fit to the distribution is overplotted as a red line. Inset: expanded plot of the distribution around the peak. Bottom: the observed distribution is compared to simulations derived for average magnetic fields of $100 \mathrm{G}, 200 \mathrm{G}$, and $300 \mathrm{G}$, respectively.

The simulations allow us to study the effect of changing the net magnetic flux density on solar granulation and how this modifies the properties of MBPs. To produce a theoretical distribution of their area, we first computed a series of consecutive $G$-band snapshots, based upon different average magnetic flux densities. The same procedures for MBP detection and area estimation were then applied directly to these simulations. We utilized 100, 200, and $300 \mathrm{G}$ signed vertical magnetic flux simulations and compared the resulting distributions directly with the observations. The spatial resolution of simulations has not been degraded (Figure 4). All three distributions, based on different initial magnetic flux densities, agree with the log-normal form of the observed MBP area distribution. Crucially, these simulated $G$-band images also exhibit the same sharp decline from the peak to the diffraction limit. The peak of the distributions does not alter significantly, with a simulated maximum at $\approx 50,000 \mathrm{~km}^{2}$. The simulations were degraded by re-binning the data to match the observational scale of $50 \mathrm{~km} \mathrm{pixel}^{-1}$. 
A further comparison revealed that the simulated distribution remained unchanged.

\section{CONCLUDING REMARKS}

We utilize an improved automatic detection algorithm to study the area distribution of solar MBPs. The area distribution of MBPs follows log-normal statistics. We interpret this result in a similar fashion as Bogdan et al. (1988), who suggest that the underlying fragmentation process is responsible for log-normal distributions. Similarly, we cannot rule out coalescence.

The peak of our MBP distribution occurs at $45,000 \mathrm{~km}^{2}$ significantly higher than our telescope diffraction limit and is consistent with the results of the radiative MHD simulations. The minimal area of MBPs is most likely defined by the width of the inter-granular lanes, which is subsequently limited by the radiative and convective energy balance, and mass conservation in magneto-convective processes. The peak in the area distribution seems to correspond to the most probable width of the inter-granular lane. The area of large MBPs may be limited by the lack of sufficient radiative heating in the larger flux tubes. As has been demonstrated (see, e.g., Berger et al. 1995), wall heating of magnetic flux concentrations is not sufficient to increase the vertical radiative flux for flux tubes greater than $500 \mathrm{~km}$ in width. Thus, MBPs cannot be generated in large diameter magnetic flux tubes. Elongated bright points of large size may still, in principle, be formed. However, their formation will be inhibited by strong plasma motions and granule fragmentation.

This work has been supported by the UK Science and Technology Facilities Council (STFC). Observations were obtained at the National Solar Observatory, operated by the Association of Universities for Research in Astronomy, Inc. (AURA), under cooperative agreement with the National Science Foundation. P.J.C. thanks the Northern Ireland Department for Employment and Learning for a PhD studentship. D.B.J. is grateful to STFC for the award of a post-doctoral fellowship. F.P.K. is grateful to AWE Aldermaston for the award of a William Penney Fellowship. We wish to thank the anonymous referee for useful comments and suggestions.

\section{REFERENCES}

Berger, T. E., Schrijver, C. J., Shine, R. A., Tarbell, T. D., Title, A. M., \& Scharmer, G. 1995, ApJ, 454, 531

Bogdan, T. J., Gilman, P. A., Lerche, I., \& Howard, R. 1988, ApJ, 327, 451

Bovelet, B., \& Wiehr, E. 2001, Sol. Phys., 201, 13

Bovelet, B., \& Wiehr, E. 2003, A\&A, 412, 249

Caunt, S. E., \& Korpi, M. J. 2001, A\&A, 369, 706

Crockett, P. J., Jess, D. B., Mathioudakis, M., \& Keenan, F. P. 2009, MNRAS, 397, 1852

de Wijn, A. G., Stenflo, J. O., Solanki, S. K., \& Tsuneta, S. 2009, Space Sci. Rev., 144, 275

Ishikawa, R., et al. 2007, A\&A, 472, 911

Jess, D. B., Mathioudakis, M., Christian, D. J., Keenan, F. P., Ryans, R. S. I., \& Crockett, P. J. 2010, Sol. Phys., 261, 363

Jess, D. B., Mathioudakis, M., Crockett, P. J., \& Keenan, F. P. 2008, ApJ, 688, L119

Langhans, K., Schmidt, W., \& Tritschler, A. 2002, A\&A, 394, 1069

Sánchez Almeida, J., Asensio Ramos, A., Trujillo Bueno, J., \& Cernicharo, J. 2001, ApJ, 555, 978

Sánchez Almeida, J., Márquez, I., Bonet, J. A., Domínguez Cerdeña, I., \& Muller, R. 2004, ApJ, 609, L91

Schüssler, M., Shelyag, S., Berdyugina, S., Vögler, A., \& Solanki, S. K. 2003, ApJ, 597, L173

Shelyag, S., Fedun, V., \& Erdélyi, R. 2008, A\&A, 486, 655

Shelyag, S., Schüssler, M., Solanki, S. K., Berdyugina, S. V., \& Vögler, A. 2004, A\&A, 427, 335

Sod, G. A. 1978, J. Comput. Phys., 27, 1

Steiner, O., Hauschildt, P. H., \& Bruls, J. 2001, A\&A, 372, L13

Title, A. M., \& Berger, T. E. 1996, ApJ, 463, 797

Utz, D., Hanslmeier, A., Möstl, C., Muller, R., Veronig, A., \& Muthsam, H. 2009, A\&A, 498, 289

Vögler, A., Shelyag, S., Schüssler, M., Cattaneo, F., Emonet, T., \& Linde, T. 2005, A\&A, 429, 335

Wall, J. V., \& Jenkins, C. R. 2003, Princeton Series in Astrophysics (Cambridge: Cambridge Univ. Press)

Wiehr, E., Bovelet, B., \& Hirzberger, J. 2004, A\&A, 422, L63

Wöger, F., von der Lühe, O., \& Reardon, K. 2008, A\&A, 488, 375 